\title{
BMJ Open Outcomes evaluating quality of life and their measurement properties in early- onset scoliosis: protocol for a systematic review
}

\author{
Charles Baird (D) , ${ }^{1}$ James Archer, ${ }^{1}$ Adrian Gardner, ${ }^{1}$ Alison B Rushton (D) , ${ }^{2}$ \\ Nicola R Heneghan (iD ${ }^{3}$
}

To cite: Baird C, Archer J, Gardner A, et al. Outcomes evaluating quality of life and their measurement properties in early-onset scoliosis: protocol for a systematic review. BMJ Open 2021;11:e048956. doi:10.1136/ bmjopen-2021-048956

- Prepublication history and additional supplemental material for this paper are available online. To view these files, please visit the journal online (http://dx.doi.org/10.1136/ bmjopen-2021-048956)

Received 11 January 2021 Accepted 17 August 2021

D) Check for updates

C Author(s) (or their employer(s)) 2021. Re-use permitted under CC BY-NC. No commercial re-use. See rights and permissions. Published by BMJ.

${ }^{1}$ Royal Orthopaedic Hospital NHS Foundation Trust, Birmingham, UK

${ }^{2}$ School of Physical Therapy, Western University Faculty of Health Sciences, London, Ontario, Canada

${ }^{3}$ School of Sport, Exercise and Rehabilitation Sciences, University of Birmingham, Birmingham, UK

Correspondence to Professor Adrian Gardner; adrian.gardner@nhs.net

\section{ABSTRACT}

Introduction Early-onset scoliosis (EOS) is a rare spinal deformity affecting children under the age of 10 . Both the condition and its treatment have associated morbidity and can impact quality of life. Understanding this impact can be achieved by using appropriate patient-reported and/or carer-reported outcome measures. The aim of the review described in this protocol is to evaluate the evidence on measurement properties relevant to health-related quality of life outcomes in the EOS population. The focus will be on outcome measures relevant to patients undergoing treatment of EOS under the age of 10.

Methods/analysis This protocol is reported in line with Preferred Reporting Items for Systematic Reviews and Meta-Analyses Protocol and COnsensus-based Standards for the selection of health Measurement Instruments (COSMIN) methodology. The MEDLINE, EMBASE, EMCARE, PubMed, PsychINFO and CINAHL databases will be searched using a two-stage search strategy. The first stage will identify measures of HRQOL used in EOS through screening of titles and abstracts. The second stage will assess the measurement properties of those measures identified through screening of full-text articles. The measurement properties of interest are the 'reliability', 'validity' and 'responsiveness' of the instrument. Only English language articles will be considered. Two reviewers will independently review the search results against the eligibility criteria, perform data extraction and assess for risk of bias, with disputes handled by a third reviewer. Data will be quantitatively pooled where possible or reported as a narrative synthesis. The summarised results for each measurement property will be rated against the criteria for good measurement properties following the COSMIN methodology. Two reviewers will assess the body of evidence for each measurement property using modified Grading of Recommendations, Assessment, Development and Evaluation guidelines.

Ethics and dissemination No ethical approval is required for this review and the results will be submitted for publication in peer-reviewed publications. PROSPERO registration number CRD42020219721.

\section{INTRODUCTION}

Scoliosis is a three-dimensional rotational alteration in the normal shape of the spine,
Strengths and limitations of this study

- A two-stage search strategy will be used to identify current measures of health-related quality of life in early-onset scoliosis and then identify evidence assessing their measurement properties.

- The protocol has been designed in line with the COnsensus-based Standards for the selection of health Measurement INstruments methodology and evidence will be rated as per a modified Grading of Recommendations, Assessment, Development and Evaluation approach.

- Strengths of the proposed methodology a two-stage search approach and the use of two independent reviewers for data extraction and analysis.

- A limitation of the review is its exclusivity to Englishlanguage studies.

defined by a Cobb angle of greater than $10^{\circ}$ in the coronal plane. ${ }^{1}$ When this is diagnosed before the age of 10 , it is classified as earlyonset scoliosis (EOS). ${ }^{2}$ EOS is a rare, heterogeneous condition of variable severity with multiple underlying causes and is associated with a number of medical conditions. A classification based on aetiology has been proposed by Williams $e t a l^{3}$ comprising four categories of EOS: congenital (due to a congenital vertebral abnormality), neuromuscular (occurring secondary to an underlying neuromuscular disorder), syndromic (in association with a broader systemic syndrome) and idiopathic (of unknown cause). The estimated prevalence of EOS in the USA is in the range of 4-10 cases per 10000 children. $^{4}$

Untreated, a severe spinal curvature in a young child impairs cardiac and pulmonary development, predisposing to premature cardiorespiratory failure. ${ }^{56}$ This carries an increased risk of mortality by the age of 40 , or earlier in more severely affected children. ${ }^{7}$ The curvature may also impair a patient's 
physical function and cause pain and disability. ${ }^{8}$ Additionally, the financial and caregiver burden for patients with EOS is reported to be greater than that of healthy aged-matched peers. ${ }^{10}$

The goals of management of EOS include maximising lung function, spinal growth and mobility, while minimising the spinal curvature and the extent of any required fusion procedure. ${ }^{11}$ Conservative management is appropriate in a subset of patients with a resolving idiopathic deformity. ${ }^{12}$ Progressive curves require treatment with bracing, casting or surgical intervention. ${ }^{13}$ Management by any method often takes many years and may require multiple hospital visits and interventions.

Implicit within the management goals is the improvement of the health-related quality of life (HR-QoL) of patients. HR-QoL is a broad, multidimensional concept composed of physical, psychological, social and environmental domains, representing the 'well-being' of an individual or group. ${ }^{14}$ An individual or group's 'well-being' is related to their level of 'functioning' or 'disability' with regard to each of these domains. This may be better understood using the International Classification of Functioning, Disability and Health (ICF) conceptual framework. ${ }^{1516}$ This framework identifies that it is the 'impairments', 'activity limitations' and 'participation restrictions' experienced by an individual or group that constitute their level of functioning or disability and affect their QoL. The ICF additionally clarifies that these restrictions and limitations cannot be assumed based solely on the existence of a medical condition, emphasising a shift in focus from the diagnosis to an evaluation of functioning and life experience.

Due to the multifactorial nature of the life of any individual, the evaluation and measurement of the life experience of any specific patient (HR-QoL) is complicated. It is commonly performed through administering one or multiple generic or disease-specific questionnaires. ${ }^{17} 18$ Measuring HR-QoL in patients with EOS is challenging due to the requirement to use age-appropriate patientreported outcome measures (PROM), the ability of paediatric patients to self-report and the heterogeneity and variable severity of coexistent health conditions (eg, muscular dystrophy, cerebral palsy, trisomy 21) seen in some of the children. Assessment often requires the use of PROM and/or carer-reported outcome measures (CROMs). As yet there is no standardised HR-QoL measure (forming part of a 'core outcome set' as per the COMET initiative) ${ }^{19}$ for the EOS population.

Instruments measuring HR-QoL should have adequate measurement properties to ensure that within the HR-QoL the views of that particular individual are reflected as closely as possible. The COnsensus-based Standards for the selection of health Measurement INstruments (COSMIN) group have defined desirable measurement properties, identifying 'reliability', 'validity' and 'responsiveness' of an outcome measure as key domains. ${ }^{20}$ The COSMIN group have further expanded the taxonomy of measurement properties, to include the instrument's
Box 1 The COSMIN taxonomy of measurement property terms (as specified in the COSMIN guideline) ${ }^{20}$

Measurement properties
Content validity
PROM Development
Content validity
Internal structure
Structural validity
Internal consistency
Cross-cultural validity Imeasurement invariance
Remaining measurement properties
Reliability
Measurement error
Criterion validity
Hypotheses testing for construct validity
CoSMIN, COnsensus-based Standards for the selection of health Measurement
Instruments; PROM, patient-reported outcome measure.

'interpretability' and 'feasibility' along with additional subcategories, listed in box 1. Evaluating measures of HR-QoL with regard to these measurement properties is necessary to understand overall instrument performance and in the selection of the best measure(s).

Assessing HR-QoL in patients with EOS is particularly relevant given the introduction of new surgical strategies, including growth guidance, which have been designed to reduce the operative burden of treatment. ${ }^{21-23}$ Additionally, the James Lind Alliance identified that understanding how QoL is affected by scoliosis and how this can be measured was one of the top 10 priorities in scoliosis research in $2017 .{ }^{24}$ A review is therefore justified to establish current understanding of QoL assessment in children with EOS.

\section{Aims of review}

To evaluate the evidence relevant to HR-QoL assessment in patients with EOS, specifically those patients under the age of 10 years undergoing bracing, surgery or conservative treatment. The first objective will be to identify relevant outcome measures. The second objective will be to evaluate the measurement properties of those identified instruments.

\section{METHODS}

This protocol has been devised following collaboration between experts in musculoskeletal rehabilitation research, physiotherapy and scoliosis. It has been designed in line with the COSMIN methodology for systematic reviews of patient-reported outcomes. ${ }^{20}$ The protocol is reported in line with the Preferred Reporting Items for Systematic Reviews and Meta-Analyses (PRISMA) Proto$\operatorname{cols}^{25}$ (online supplemental file 1).

The proposed methodology has a two-stage approach. In stage 1, broad searches will be conducted to identify what specific instruments or outcome measures 
are used in contemporary and historic literature to measure HR-QoL in patients with EOS. In stage 2, searches will be conducted for studies evaluating the measurement properties of the instruments that were identified in stage 1.

\section{Stage 1: identifying measures of HR-QoL \\ Eligibility criteria \\ Participants}

Participants less than 10 years of age with a diagnosis of scoliosis and Cobb angle of $>10^{\circ}$ will be considered (as per the diagnostic criteria for EOS).$^{2}$ No restrictions will be applied to the associated medical conditions, curve severity or treatment modality.

\section{Outcome}

Any study that includes assessments of HR-QoL involving a PROM or CROM will be included. As per the ICF conceptual framework, HR-QoL pertains to the 'activity limitation', 'participation restriction' and 'impairments' experienced by an individual. ${ }^{15} 16$

\section{Study design}

All study designs including randomised clinical trials, cohort, observational studies and case studies will be included to identify all PROM of HR-QoL used in individuals with $\mathrm{EOS}$.

No limitation on geographical location.

\section{Search strategy}

The strategy has been informed by scoping searches and discussions with experts (methodological, subject specific and a medical librarian) and will involve systematic searches of electronic databases with structured search blocks. The search will be completed by one reviewer (CB). The search blocks in the first stage will contain terms relevant to the following:

- Population of interest: patients with EOS.

- Construct of interest: HR-QoL.

An example of the search strategy and actual search terms to be used is included in online supplemental file 1 . Search results will be filtered for participants of the appropriate age (less than 10) where this software function is available. The title and abstracts of the eligible studies will be independently reviewed by two authors ( $\mathrm{CB}$ and JA) and the PROM used in the studies to evaluate the construct of interest (HRQoL) recorded. Following stage 1, it is anticipated that a number of PROMs will have been identified. Multiple uses of the same PROM will be tallied, and the full name of the tool as well as the abbreviated reference to the tool will be extracted for use in the stage 2 search. The PROQOLID database, which is a free access, online database that acts as a repository and index of QoL instruments, will be searched separately for instruments used or deemed appropriate for use in EOS.
Stage 2: evaluating the measurement properties of the identified PROM

Eligibility criteria

\section{Participants}

Participants up to 10 years of age with a diagnosis of scoliosis and a Cobb angle of $>10^{\circ}$ will be eligible. In studies of mixed cohorts, more than $50 \%$ of participants should be individuals with EOS. There will be no exclusion of studies based on disease severity or treatment modality (conservative/bracing/surgery) of the study cohort.

\section{Outcome}

The outcomes of interest are the measurement properties of the identified instrument, including reliability (internal consistency, test-retest, inter-rater and intrarater), measurement error, validity (content validity, structural validity or criterion validity), hypothesis testing and responsiveness as per the COSMIN taxonomy. ${ }^{20}$

\section{Study design}

Any study evaluating one or more measurement properties of the PROM, identified in search 1, including development and validation studies will be included. Studies where the design is not focused to evaluate the instrument measurement properties or where the instrument/PROM is used in a validation study of another instrument will be excluded, as per the COSMIN methodology. ${ }^{20}$ In the event that groups of tools have been compared and the distinction between reference and test tools is not clear, authors will be contacted for clarification. If clarification is not possible, then this will be reported transparently. Studies on instrument responsiveness will be included where this is evaluated based on hypothesis testing of expected treatment effect (before and after intervention) or comparison of subgroups of disparate severity (eg, minor curve idiopathic vs major curve neuromuscular). This is as recommended in the COSMIN methodology in the absence of a gold standard. ${ }^{20}$ Studies where a fulltext English language publication is not available will be excluded. Studies of English-language versions of tools will be included. Conference abstracts will be excluded. Studies without original participant data (eg, systematic review) will be excluded.

Authors of studies will be contacted in case of missing information.

\section{Search strategy}

Searches of electronic databases will be conducted using structured search blocks in order to identify studies evaluating measurement properties of each instrument identified in stage 1 . The search will be completed by one reviewer (CB). A search will be conducted for each instrument using search blocks containing terms relevant to the following:

- Population of interest: patients with EOS.

- Measurement instrument: (identified in stage 1).

- Measurement properties filter. ${ }^{26}$

- Exclusion filter. ${ }^{26}$ 
The measurement property and exclusion filter will use search blocks recommended in the COSMIN methodology from Terwee et al..$^{26}$ For efficiency, all measurement instruments will be included in a single search block, each term separated by 'OR'. An example of the search strategy and actual search terms to be used is included in online supplemental file 2 .

\section{Information sources}

The electronic records of the NHS Open Athens healthcare databases will be searched. This includes CINAHL (1937-December 2020), EMBASE (1974-December 2020), EMCARE (1995-December 2020), Medline (1946December 2020), PsycINFO (1967-December 2020) and PubMed (1997-December 2020). The rationale for searching PubMed in addition to MEDLINE is to access 'ahead of print' or 'in process' articles. The PROQOLID database, an online database of QoL instruments, will be also searched for instruments used or deemed appropriate for use in EOS.

\section{Data management}

Search records will be imported into Mendeley Reference Management software (London, UK) and the web-based systematic review app Rayyan QCRI (Dohar, Qatar). ${ }^{27}$ Duplicates will be identified and excluded in Rayyan QCRI. Rayyan will also be used to identify reviewer dispute, facilitate third party (AG) dispute resolution and tally study inclusion and exclusion.

\section{Study selection process}

Eligibility of the articles at each stage will be determined by two authors (CB and JA) independently by reviewing the article title and abstract against the eligibility criteria. If the title or abstract are insufficient to determine eligibility then full-text versions will be requested. A third author (AG) will be involved to resolve eligibility disputes. A PRISMA flow diagram will be constructed to allow transparency over the inclusion and exclusion of studies.

\section{Data collection process}

This will be conducted independently by two authors (CB and JA) and data will be tabulated in an 'overview table' format similar to that suggested in the COSMIN methodology. Any disagreements between reviewers will be mediated through discussion with a third reviewer (AG). Examples of the tables to be used for data extraction are appended in online supplemental file 3 and are similar to those recommended in the COSMIN guideline.

\section{Data items}

A summary of the data items to be extracted from each study is shown in table 1 .

\section{Risk of bias in individual studies}

The COSMIN Risk of Bias checklist will be used to assess methodological quality in individual studies, determine which measurement properties (as per the COSMIN taxonomy and definitions-box 1) are being assessed in each study and facilitate the extraction of further data items relevant to methodological analysis (table 1). ${ }^{20}$ Subjective judgement may be necessary at this stage regarding the terms and definitions used in each study as these may not be similar to the COSMIN taxonomy. It is also possible that multiple measurement properties may be explored in a single study, and in this case, each assessment of a measurement property will be appraised separately. The questions within the Risk of Bias checklist may not apply to all studies and only those appropriate to the focus of the paper will be used (eg, internal consistency evaluation will not be appraised in a paper focusing on content validity).

\section{Table 1 Summary of data items to be extracted from the included studies}

Study and participants characteristics

Outcome measure

Measurement properties
Reference, year, country, design of study, age, gender, sample size (used in the analysis), type of intervention (including but not limited to casting, traditional growing rods, magnetic growing rods, VEPTR (Vertical Expansile Prosthetic Titanium Rib), Shilla, Tether), diagnostic subgroups of participants (congenital/idiopathic/syndromic/neuromuscular), curve severity and curve pattern

Name of outcome measure, version of outcome measure, means of scores, mode of administration, recall period, subscale, numbers of items, response option, response rate, missing items, setting, target population, scoring, original language, available translation

Validity : Type of validity, descriptive statistics, missing value, comparator outcome or predictor outcome, hypothesis, statistics methods (including IRT/CTT), Cl, validation results, sample size

Reliability : Type of reliability, descriptive statistic, time interval, reliability coefficient, measurement error, sample size, number of repeated measurements

Responsiveness: Method of testing : hypothesis testing versus distribution based (ES, SRM and MDC) vs anchorbased (MIC or MCIC or MID), time to follow-up, curve severity at baseline and follow-up, curve aetiology, treatment modality

Interpretability : Distribution of score in the study population, percentage of missing items, floor and ceiling effects, scores and change scores available for relevant (sub)groups, information on response shift

Feasibility : Patient's comprehensibility, clinician's comprehensibility, type and ease of administration, length of instrument, completion time, patient's required mental and physical ability level, ease of standardisation, ease of score calculation, cost of instrument, required equipment, availability in different settings, regulatory agency's requirement for approval

CTT, classical test theory; ES, effects size; IRT, item-response theory; MCIC, minimal clinically important change; MDC, minimal detectable change; MIC, minimal important change; MID, minimal important difference; SRM, standardised response mean. 
As per COSMIN methodology, a four-point rating system will be used to rate the methodological quality of the assessment of the denoted measurement properties outlined in box 1 . The four-point scale will be 'very good', 'adequate', 'doubtful' or 'inadequate'. The rating will be determined based on the criteria specified in the COSMIN Risk of Bias checklist. ${ }^{20}$ Ratings will be determined by two authors (CB and JA) independently, with disputes resolved through discussion or involvement of a third author $(\mathrm{AG})$. The agreement between reviewers will be reported with percentage agreement and the kappa statistic using SPSS for Windows statistical software package (IBM SPSS Statistics V.25).

The overall rating of the methodological quality of each measurement property analysis will be determined by taking the lowest rating of any standard, as per the COSMIN methodology. ${ }^{20}$ The overall ratings of the approach taken for measurement property analysis will subsequently used to grade the quality of evidence.

\section{Data synthesis}

The COSMIN guidelines for systematic reviews will be followed for synthesis of the results. ${ }^{20}$ Data on the characteristics of the PROM, its measurement properties and its interpretability and feasibility will be presented in an overview table. Measurement properties will be evaluated against the 'updated criteria for good measurement properties' and rated as either 'sufficient', 'insufficient' or 'indeterminate' (as per the COSMIN methodology). ${ }^{20}$ The 'updated criteria for good measurement properties' offer specific guidance for each measurement property in order to provide these ratings. Following completion of the overview tables, the results of different studies on each measurement property per PROM will then be compared. If studies exhibit sufficient clinical and methodological homogeneity then the results will be pooled per measurement property per tool. Quantitative pooling will be performed only when the data regard patients with comparable disease (eg, similar curve severity (Cobb angles $0^{\circ}-29^{\circ}, 30^{\circ}-50^{\circ},>50^{\circ}$ ) and the same underlying aetiological classification (idiopathic, neuromuscular, congenital, syndromic)) who have undergone comparable treatments (ie, surgical cohorts will not be pooled with non-surgical cohorts) and where responses were retrieved over similar follow-up intervals. From scoping searches, authors anticipate that the data will not be amenable to quantitative pooling and a narrative synthesis of the results will be necessary. The summarised results will be used to determine whether overall the measurement properties of the PROM are sufficient, insufficient, inconsistent or indeterminate, as per the COSMIN methodology. ${ }^{20}$ If appropriate, subgroup analysis will be carried out by age, sex, self-report versus proxy report, diagnosis or diagnostic category, treatment received and responsiveness over predefined follow-up durations of a similar length.

The recommendation of a PROM will depend on the tool's measurement properties, interpretability and feasibility. As per the COSMIN guideline, a tool will only be recommended if there is sufficient content validity and at least low quality evidence for sufficient internal consistency. ${ }^{20}$

\section{Confidence in cumulative evidence}

The quality of evidence will be graded using a Grading of Recommendations, Assessment, Development and Evaluation (GRADE) approach, modified for the evaluation of measurement properties of PROM. ${ }^{20} 28$ The GRADE approach uses five factors-risk of bias, inconsistency, indirectness, imprecision and publication bias-to produce a quality of evidence rating of either high, moderate, low or very low. As per the COSMIN methodology, publication bias will not be assessed in this review. Risk of bias will be assessed using the COSMIN risk of bias checklist. ${ }^{20}$ Where inconsistency of results across studies is identified, and results can be neither pooled nor summarised, the conclusion will be based on the majority of consistent results but the quality of evidence downgraded for inconsistency. Imprecision will be evaluated based on total sample size across studies and will be downgraded if the total sample size is less than 100 or downgraded two levels if less than 50 , as per the COSMIN guidance. ${ }^{20}$ Indirectness will be evaluated based on the degree to which studies are performed on the population of interest, and downgraded where the population of interest only form part of the study group.

Grading of evidence will be performed by two reviewers independently $(\mathrm{CB}$ and $\mathrm{JA})$ with disputes resolved by a third reviewer (AG).

\section{Patient and public involvement}

Patients and members of the public will not be consulted in the production of this research. Findings from the review will be disseminated publicly in peer-reviewed journals.

\section{DISCUSSION AND IMPLICATIONS}

The primary goal in the management of EOS is to reduce the cardiorespiratory morbidity associated with the condition through the control of the spinal curvature while allowing continued growth of the spine and thorax. ${ }^{6913}$ Implicit within, and in addition to this goal is the improvement in the HR-QoL of the patients. Clinicians, however, recognise that both the condition and management are associated with morbidity and affect patients' life experience. ${ }^{30}$ Understanding the impact of both is relevant to clinical practice and research in the condition. A review to understand the current state of the art of HR-QoL assessment in EOS is therefore justified, and this protocol aims to provide a framework for a comprehensive overview of the currently available PROM/CROMs assessing QoL and to appraise the quality of the evidence base for their measurement properties. The authors expect 
that this work will benefit clinicians and researchers in identifying whether currently available tools are appropriate for assessing HR-QoL in their patients. This review addresses a scoliosis research priority and could provide a population-specific research agenda. ${ }^{24}$

\section{Ethics}

No ethics approval is required for this systematic review. The results of the review will be disseminated through peer-reviewed journals as well as in conference presentation at national and international societies including the Scoliosis Research Society and the International Congress on EOS. Patient consent is not required for the research or publication.

Twitter Alison B Rushton @abrushton and Nicola R Heneghan @HeneghanNicola

Contributors All authors conceptualised and designed the protocol. CB drafted the manuscript. JA reviewed the manuscript. $A G, A B R$ and NRH reviewed the manuscript and provided guidance on design, topic, methodology and analysis. All authors reviewed and commented on each draft of the protocol. All authors have approved and contributed to the final manuscript.

Funding The authors have not declared a specific grant for this research from any funding agency in the public, commercial or not-for-profit sectors.

Competing interests None declared.

Patient consent for publication Not required.

Provenance and peer review Not commissioned; externally peer reviewed.

Supplemental material This content has been supplied by the author(s). It has not been vetted by BMJ Publishing Group Limited (BMJ) and may not have been peer-reviewed. Any opinions or recommendations discussed are solely those of the author(s) and are not endorsed by BMJ. BMJ disclaims all liability and responsibility arising from any reliance placed on the content. Where the content includes any translated material, BMJ does not warrant the accuracy and reliability of the translations (including but not limited to local regulations, clinical guidelines, terminology, drug names and drug dosages), and is not responsible for any error and/or omissions arising from translation and adaptation or otherwise.

Open access This is an open access article distributed in accordance with the Creative Commons Attribution Non Commercial (CC BY-NC 4.0) license, which permits others to distribute, remix, adapt, build upon this work non-commercially, and license their derivative works on different terms, provided the original work is properly cited, appropriate credit is given, any changes made indicated, and the use is non-commercial. See: http://creativecommons.org/licenses/by-nc/4.0/.

\section{ORCID iDs}

Charles Baird http://orcid.org/0000-0001-6351-7208

Alison B Rushton http://orcid.org/0000-0001-8114-7669

Nicola R Heneghan http://orcid.org/0000-0001-7599-3674

\section{REFERENCES}

1 Kane WJ. Scoliosis prevalence: a call for a statement of terms. Clin Orthop Relat Res 1977;126:43-6 https://pubmed.ncbi.nlm.nih.gov/ 598138/

2 Skaggs D, Guillaume T E-H. Early onset scoliosis consensus statement, SRS growing spine committee spine deformity, 2015. Available: https://www.srs.org/about-srs/quality-and-safety/positionstatements/early-onset-scoliosis-consensus-statement

3 Williams BA, Matsumoto $\mathrm{H}$, McCalla DJ, et al. Development and initial validation of the classification of early-onset scoliosis (C-EOS). $J$ Bone Joint Surg Am 2014;96:1359-67.

4 The Burden of Musculoskeletal Diseases in the United States. Spinal deformity. Available: https://www.boneandjointburden.org/fourthedition/iib0/spinal-deformity [Accessed 07 Jan 2021].
5 Davies G, Reid L. Effect of scoliosis on growth of alveoli and pulmonary arteries and on right ventricle. Arch Dis Child 1971;46:623-32.

6 Redding G, Song K, Inscore S, et al. Lung function asymmetry in children with congenital and infantile scoliosis. Spine $J$ 2008;8:639-44.

7 Pehrsson K, Larsson S, Oden A, et al. Long-term follow-up of patients with untreated scoliosis. a study of mortality, causes of death, and symptoms. Spine 1992;17:1091-6.

8 Weinstein SL, Dolan LA, Spratt KF, et al. Health and function of patients with untreated idiopathic scoliosis: a 50-year natural history study. JAMA 2003;289:559-67.

9 Fernandes P, Weinstein SL. Natural history of early onset scoliosis. $J$ Bone Joint Surg Am 2007;89 Suppl 1:21-33.

10 Campbell M, Matsumoto $\mathrm{H}$, St Hilaire T, et al. Burden of care in families of patients with early onset scoliosis. $J$ Pediatr Orthop $B$ 2020;29:567-71.

11 Scoliosis Research Society. Early onset scoliosis. Available: https:// www.srs.org/patients-and-families/conditions-and-treatments/ parents/scoliosis/early-onset-scoliosis [Accessed 07 Jan 2021].

12 Ferreira JH, de Janeiro R, James JIP. Progressive and resolving infantile idiopathic scoliosis. J Bone Joint Surg Br 1972;54B:648-55.

13 Helenius IJ. Treatment strategies for early-onset scoliosis. EFORT Open Rev 2018;3:287-93.

14 World Health Organisation. Whoqol: measuring quality of life. Available: https://www.who.int/healthinfo/survey/whoqol-qualityoflife/ en/ [Accessed 19 Jun 2020].

15 World Health Organization. ICF. International classification of functioning, disability and health. Geneva, 2001.

16 McDougall J, Wright V, Rosenbaum P. The ICF model of functioning and disability: incorporating quality of life and human development. Dev Neurorehabil 2010;13:204-11.

17 Guyatt GH, Feeny DH, Patrick DL. Measuring health-related quality of life. Ann Intern Med 1993;118:622-9.

18 Bagó J, Climent JM, Pérez-Grueso FJS, et al. Outcome instruments to assess scoliosis surgery. Eur Spine $J$ 2013;22:195-202.

19 Dodd S, Clarke M, Becker L, et al. A taxonomy has been developed for outcomes in medical research to help improve knowledge discovery. J Clin Epidemiol 2018;96:84-92.

20 Prinsen CAC, Mokkink LB, Bouter LM. COSMIN manual for systematic reviews of PROMs COSMIN user manual, 2018: 1-78. https://www.cosmin.nl/wp-content/uploads/COSMIN-syst-reviewfor-PROMs-manual_version-1_feb-2018-1.pdf

21 Teoh KH, Winson DMG, James SH, et al. Magnetic controlled growing rods for early-onset scoliosis: a 4-year follow-up. Spine $J$ 2016;16:S34-9.

22 Hickey BA, Towriss C, Baxter G, et al. Early experience of MAGEC magnetic growing rods in the treatment of early onset scoliosis. Eur Spine $J$ 2014;23 Suppl 1:61-5.

23 Bauer JM, Yorgova P, Neiss G, et al. Early onset scoliosis: is there an improvement in quality of life with conversion from traditional growing rods to magnetically controlled growing rods? J Pediatr Orthop 2019;39:e284-8.

24 Priority 2 from the scoliosis PSP. James Lind Alliance. Available: http://www.jla.nihr.ac.uk/priority-setting-partnerships/scoliosis/ priority-2-from-the-scoliosis-psp.htm [Accessed 07 Jan 2021].

25 Moher D, Shamseer L, Clarke M. Preferred reporting items for systematic review and meta-analysis protocols (PRISMA-P) 2015 statement. Rev Esp Nutr Humana y Diet 2016;20:148-60.

26 Terwee CB, Jansma EP, Riphagen II, et al. Development of a methodological pubmed search filter for finding studies on measurement properties of measurement instruments. Qual Life Res 2009;18:1115-23.

27 Ouzzani M, Hammady H, Fedorowicz Z, et al. Rayyan-a web and mobile app for systematic reviews. Syst Rev 2016;5:210.

28 Guyatt G, Oxman AD, Akl EA, et al. Grade guidelines: 1. Introductiongrade evidence profiles and summary of findings tables. $J$ Clin Epidemiol 2011;64:383-94.

29 Balshem H, Helfand M, Schünemann HJ, Guyatt GH, et al. Grade guidelines: 3 . rating the quality of evidence. $J$ Clin Epidemiol 2011;64:401-6.

30 Phillips JH, Knapp DR, Herrera-Soto J. Mortality and morbidity in early-onset scoliosis surgery. Spine 2013;38:324-7. 